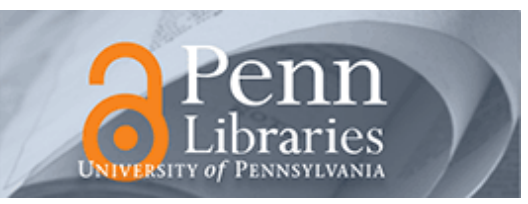

University of Pennsylvania

ScholarlyCommons

$1-1-2003$

\title{
Bandwidth, Cross-Polarization, and Feed-Point Characteristics of Matched Hilbert Antennas
}

Jinhui Zhu

Villanova University

Ahmad Hoorfar

Villanova University

Nader Engheta

University of Pennsylvania, engheta@seas.upenn.edu

Follow this and additional works at: https://repository.upenn.edu/ese_papers

Part of the Electrical and Computer Engineering Commons

\section{Recommended Citation}

Jinhui Zhu, Ahmad Hoorfar, and Nader Engheta, "Bandwidth, Cross-Polarization, and Feed-Point Characteristics of Matched Hilbert Antennas", . January 2003.

Copyright Year IEEE. Reprinted from IEEE Antennas and Wireless Propagation Letters, Volume 2, Issue 1, January 2003, pages 2-5.

Publisher URL:http://ieeexplore.ieee.org/xpl/tocresult.jsp?isNumber=27112\&puNumber=7727

This material is posted here with permission of the IEEE. Such permission of the IEEE does not in any way imply IEEE endorsement of any of the University of Pennsylvania's products or services. Internal or personal use of this material is permitted. However, permission to reprint/republish this material for advertising or promotional purposes or for creating new collective works for resale or redistribution must be obtained from the IEEE by writing to pubs-permissions@ieee.org. By choosing to view this document, you agree to all provisions of the copyright laws protecting it.

This paper is posted at ScholarlyCommons. https://repository.upenn.edu/ese_papers/11

For more information, please contact repository@pobox.upenn.edu. 


\title{
Bandwidth, Cross-Polarization, and Feed-Point Characteristics of Matched Hilbert Antennas
}

\begin{abstract}
In this work, we have applied a moment-method-based simulation code to perform a detailed parametric study of the bandwidth, cross-polarization level, and the feed location characteristics of the matched Hilbert antenna. It is shown that a properly chosen off-center near-the-end feed point may provide an approximate $50-\Omega$ real input impedance at the fundamental resonant frequency. The role of different iteration order for Hilbert antennas, approximately matched to $50-\Omega$ line, on the bandwidth and crosspolarization level of such antennas are investigated numerically. As was shown by Vinoy et al. the radiation patterns resemble those of a linear dipole and for a fixed-area-matched Hilbert antenna, the resonant frequency can be lowered by increasing the iteration order $n$. However, for such matched antennas, the bandwidth for VSWR $<2$ will be decreased and the cross-polarization level may be different for the higher iteration-order antennas.
\end{abstract}

\section{Keywords}

Bandwidth, Hilbert antenna, input impedance, matched antenna, miniaturized antenna, small antenna

\section{Disciplines}

Electrical and Computer Engineering

\section{Comments}

Copyright Year IEEE. Reprinted from IEEE Antennas and Wireless Propagation Letters, Volume 2, Issue 1, January 2003, pages 2-5.

Publisher URL:http://ieeexplore.ieee.org/xpl/tocresult.jsp?isNumber=27112\&puNumber=7727

This material is posted here with permission of the IEEE. Such permission of the IEEE does not in any way imply IEEE endorsement of any of the University of Pennsylvania's products or services. Internal or personal use of this material is permitted. However, permission to reprint/republish this material for advertising or promotional purposes or for creating new collective works for resale or redistribution must be obtained from the IEEE by writing to pubs-permissions@ieee.org. By choosing to view this document, you agree to all provisions of the copyright laws protecting it. 


\title{
Bandwidth, Cross-Polarization, and Feed-Point Characteristics of Matched Hilbert Antennas
}

\author{
Jinhui Zhu, Ahmad Hoorfar, Senior Member, IEEE, and Nader Engheta, Fellow, IEEE
}

\begin{abstract}
In this work, we have applied a moment-methodbased simulation code to perform a detailed parametric study of the bandwidth, cross-polarization level, and the feed location characteristics of the matched Hilbert antenna. It is shown that a properly chosen off-center near-the-end feed point may provide an approximate $50-\Omega$ real input impedance at the fundamental resonant frequency. The role of different iteration order for Hilbert antennas, approximately matched to $50-\Omega$ line, on the bandwidth and cross-polarization level of such antennas are investigated numerically. As was shown by Vinoy et al. the radiation patterns resemble those of a linear dipole and for a fixed-area-matched Hilbert antenna, the resonant frequency can be lowered by increasing the iteration order $n$. However, for such matched antennas, the bandwidth for VSWR $<2$ will be decreased and the cross-polarization level may be different for the higher iteration-order antennas.
\end{abstract}

Index Terms-Bandwidth, Hilbert antenna, input impedance, matched antenna, miniaturized antenna, small antenna.

\section{INTRODUCTION}

$\mathbf{S}_{\mathrm{n}}^{\mathrm{p}(\mathrm{cit})}$ PACE-FILLING curves are, in general, a continuous mapping from a normalized one-dimensional $[0,1]$ interval to a normalized two-dimensional (2-D) region $[0,1]^{2}$ passing through every point in the 2-D region. Since Peano demonstrated the construction of the first space-filling curve in 1890, many such curves have been suggested over the years [1]. One of the most widely used of such curves is the one proposed by Hilbert in 1891.

The design of small antennas with multiband characteristics has been the subject of interest in RF communities in recent years (see e.g., [2]-[8]). As shown by others (see e.g., [3]-[8]), the Hilbert curve may provide an attractive solution for miniaturization of antennas since it offers a resonant structure that may have a small footprint as one increases the step-order in iterative filling of a 2-D region [1]. However, the questions naturally arise as to how a Hilbert antenna at its fundamental resonant frequency can be matched to a feed line with a given characteristic impedance (e.g., a 50- $\Omega$ line) and how the bandwidth and cross-polarization level of such a matched antenna may vary as one increases the iteration order $n$, in order to assess the suitability of such an antenna as a matched planar resonant antenna with an electrically small footprint.

Manuscript received September 5, 2002; revised January 10, 2003.

J. Zhu and A. Hoorfar are with the Department of Electrical and Computer Engineering, Villanova University, Villanova, PA 19085 USA.

N. Engheta is with the Department of Electrical and Systems Engineering, University of Pennsylvania, Philadelphia, PA 19104 USA (e-mail: engheta@ee.upenn.edu).

Digital Object Identifier 10.1109/LAWP.2003.810765
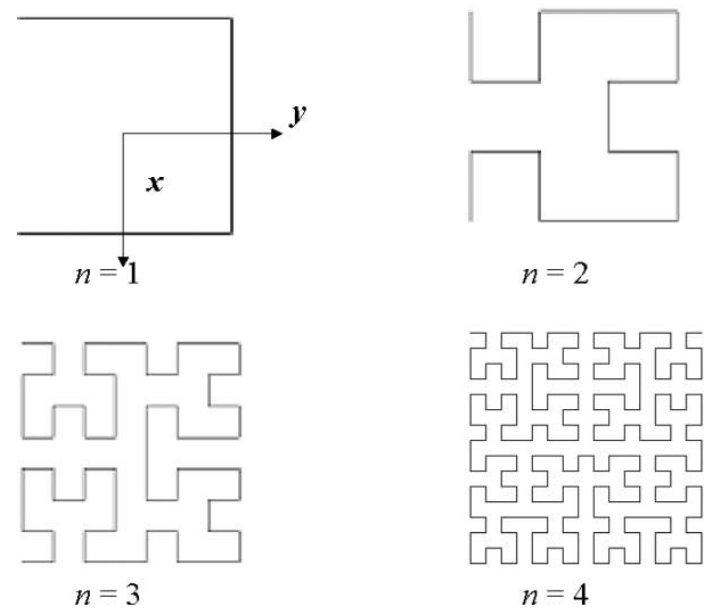

Fig. 1. Geometry of the Hilbert curves for the first four iterations. The plane $\phi=0^{\circ}$ is the $x-z$ plane.

\section{HILBERT GEOMETRY}

The Hilbert curves for the first few iterations are shown in Fig. 1. The plane-filling nature is evident by comparing the first few iteration stages. It is clear that as the iteration order increases, the total length of the line segments is increased in almost geometric progression if the side outer dimension of its "foot print" is kept fixed. For a Hilbert antenna with side dimension $L$ and order $n$, the length of each line segment $d$ and the sum of all the line segments $S$ are given by [1]

$$
d=\frac{L}{2^{n}-1} ; \quad S=\left(2^{2 n}-1\right) d=\left(2^{n}+1\right) L .
$$

One observes that the resonant frequency is decreased as the iteration order is increased (see e.g., [3]-[8]).

\section{NUMERICAL RESULTS}

In this work, we have applied the moment of method simulation code NEC-4 to perform a detailed parametric study of bandwidth variation, feed-point effects, and cross-polarization level in Hilbert antenna in free space, with no dielectric support present. We also assume that the antenna wire is perfectly conducting and, thus, no ohmic loss is considered here.

When the feed point is placed at the point of symmetry of the Hilbert antennas, the real part of the input impedance is very low at the resonant frequencies as was also shown in [3] and [4]. For example, for a third-order Hilbert antenna with side dimensions of $70 \mathrm{~mm} \times 70 \mathrm{~mm}$ [3], the real part is only about 2 $\Omega$ at the frequency where the imaginary part is zero, consistent 


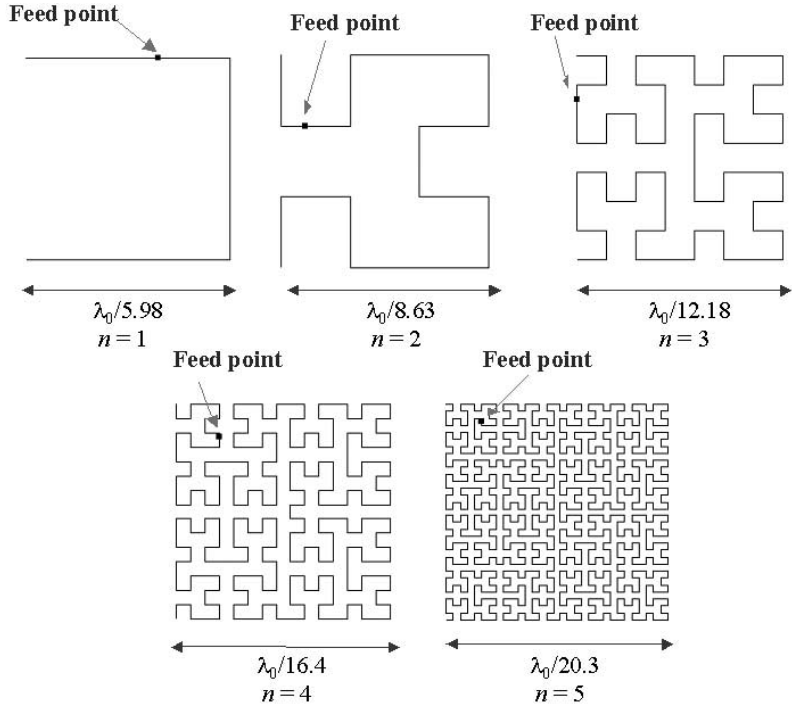

Fig. 2. The geometry and feed-point of Hilbert antennas to achieve approximately $50-\Omega$ input impedance at its fundamental resonant frequency. The relative locations of these feed points, which can be defined as the ratio of the distance along the wire from the feed point to the one end of antenna divided by the total length of the wire in the antenna $(S)$, are found to be $0.217,0.0899,0.0556,0.03628$, and 0.02786 for $n=1-5$, respectively. The normalized linear (side) dimension $\left(L / \lambda_{0}\right)$, where $\lambda_{0}$ represents the first (i.e., longest) resonant wavelength for each antenna is also shown.

TABLE I

FIRST RESONANT WPAVELENGTHS AND THE NORMALIZED TOTAL LENGTHS OF THE WIRE FOR HILBERT ANTENNAS OF ITERATION ORDER 1-5

\begin{tabular}{c|c|c|c|c|c}
\hline $\mathrm{n}$ & 1 & 2 & 3 & 4 & 5 \\
\hline$\lambda_{0}(\mathrm{~mm})$ & 418.4 & 604.1 & 852.9 & 1148.1 & 1420.7 \\
\hline$S / \lambda_{0}$ & 0.5 & 0.58 & 0.74 & 1.04 & 1.626 \\
\hline
\end{tabular}

with the results in [3]. If this antenna is fed as a monopole above the ground plane, i.e., the feed point is placed at the end of the antenna, as shown by Best [6], the input resistance is still very small at the resonant frequencies.

However, a properly chosen off-center feed point may provide, regardless of how high $n$ may be, an approximate $50-\Omega$ real input impedance at the fundamental resonant frequency of this antenna. (We have examined this up to $n=5$. We speculate that this to be approximately the case for $n>5$.) To illustrate this feature, we perform numerical simulations for Hilbert antennas of iteration orders 1-5 with fixed outer dimensions $70 \mathrm{~mm} \times 70 \mathrm{~mm}$, similar dimensions as the antennas in [3] in order to allow easy comparison of results. For each iteration order of Hilbert antenna, the location of the feed point is determined by searching for a point along the antenna for which the input impedance is about $50 \Omega$ at the first resonant frequency. In this numerical search, we start from one end of the antenna and move along the wire until we reach such a point. In these simulations, the diameter of the line is chosen to be $1 \mathrm{~mm}$. The relative location of such off-center feed points for Hilbert antennas are shown in Fig. 2 and mentioned in its caption. It is important to note that in [5] the off-center feed location has also been selected for the purpose of matching the antenna to $50-\Omega$ line. However, there the antenna is matched at its third reso-
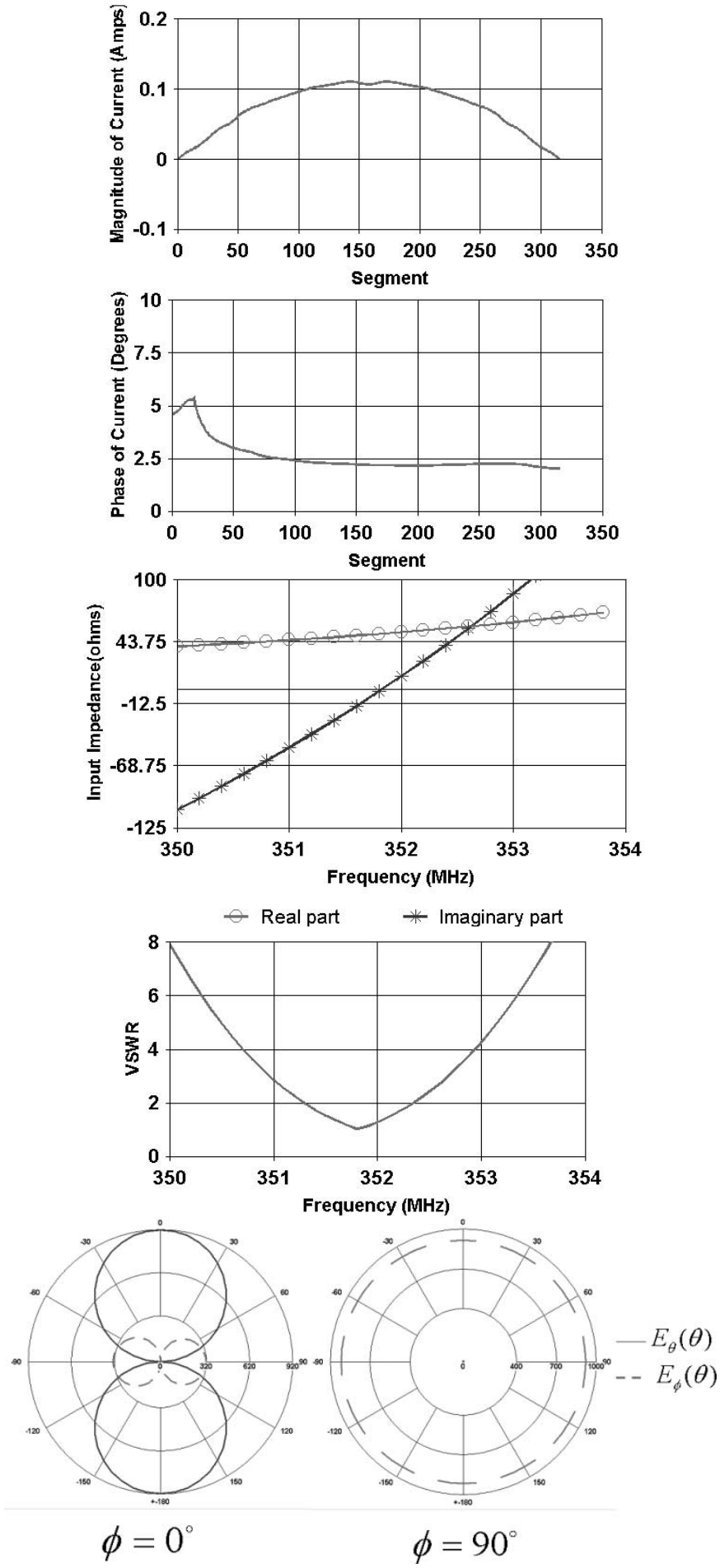

(e)

Fig. 3. Plots for the third-order off-center-fed-matched Hilbert antenna. (a) Magnitude of current distribution along the wire segments. (b) Phase of current distribution along the wire segments. (c) Real and imaginary parts of the input impedance versus frequency. (d) VSWR versus frequency. (e) The radiated electric field components in two orthogonal planes $\phi=0^{\circ}$ and $\phi=90^{\circ}$ at the lowest resonant frequency. The number of method of moments (MoM) segments (cells) we used along the wire for the numerical analysis of this antenna was 315 .

nant frequency, whereas here the matching is done for the fundamental mode, thus resulting in a different off-center location (much closer to the wire end). 


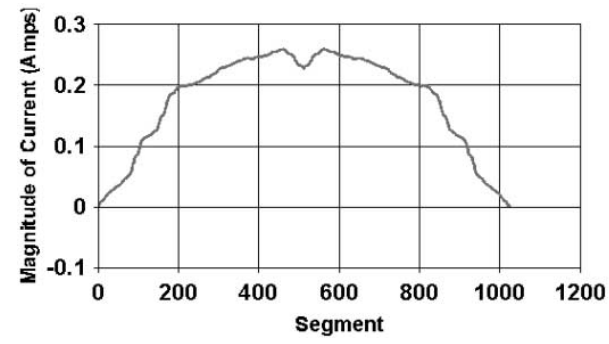

(a)

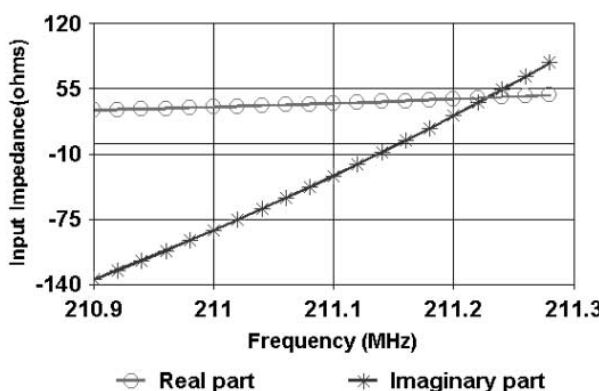

(c)

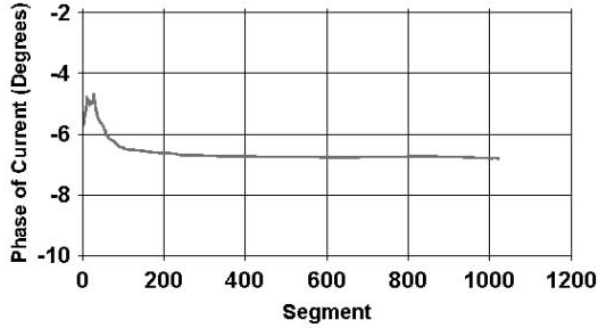

(b)

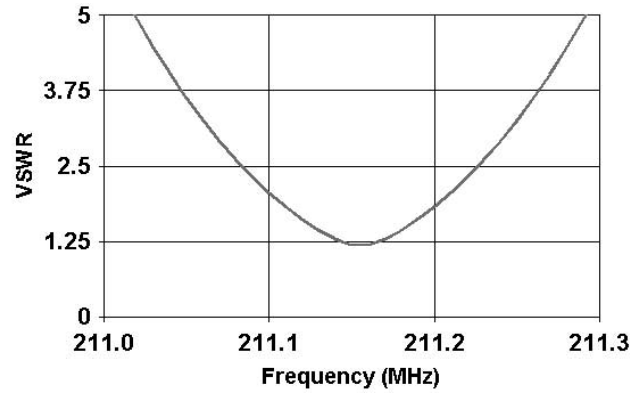

(d)

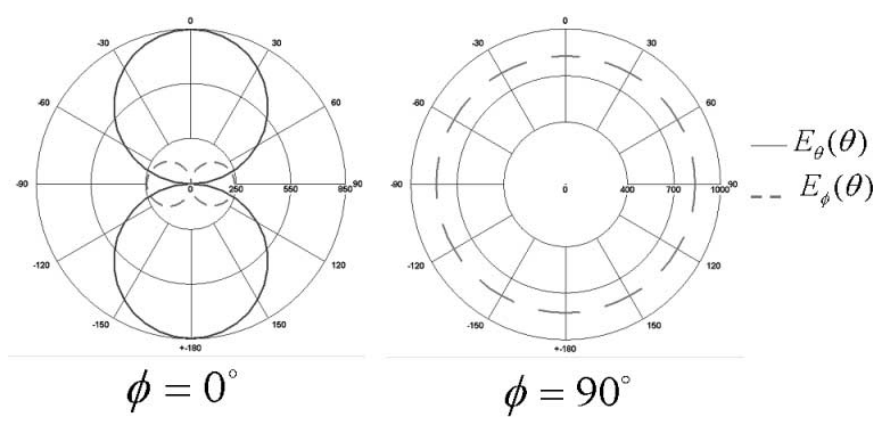

(e)

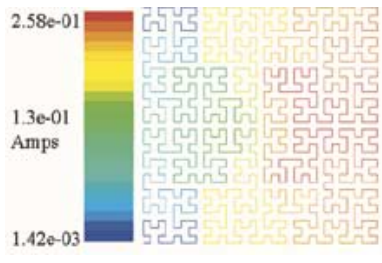

(f)

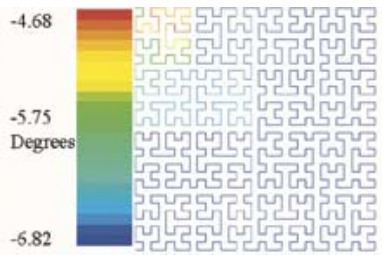

(g)

Fig. 4. Plots for the fifth-order matched Hilbert antenna. (a) Magnitude of current distribution along the wire segments. (b) Phase of current distribution along the wire segments. (c) Real and imaginary parts of the input impedance versus frequency. (d) VSWR versus frequency. (e) The radiated electric field components in two orthogonal planes $\phi=0^{\circ}$ and $\phi=90^{\circ}$ at the lowest resonant frequency. (f) Color-coded contour plot of magnitude of current distribution. (g) Color-coded contour plot of phase of current distribution. The number of MoM segments (cells) we used along the wire for the numerical analysis of this antenna was 1023.

The normalized total lengths of the wire $\left(S / \lambda_{0}\right)$ for these five Hilbert antennas are listed in Table I, where $\lambda_{0}$ denotes the first resonant wavelength (i.e., longest resonant wavelength) for each antenna. The values of $\left(L / \lambda_{0}\right)$ are shown in Fig. 2 next to their corresponding Hilbert antennas. We notice that for $n=5$, the outer dimension of the antenna is only about $1 / 20$ of its first resonant wavelength, and the antenna is still matched to $50-\Omega$ line at its fundamental resonance.

It is interesting to explore the current distribution along the wire in a Hilbert antenna. Since the current distributions, radiation patterns and multiresonant properties of the different iteration-order Hilbert antennas at resonance are somewhat similar, we just show the figures of the third and fifth iteration-order antennas.

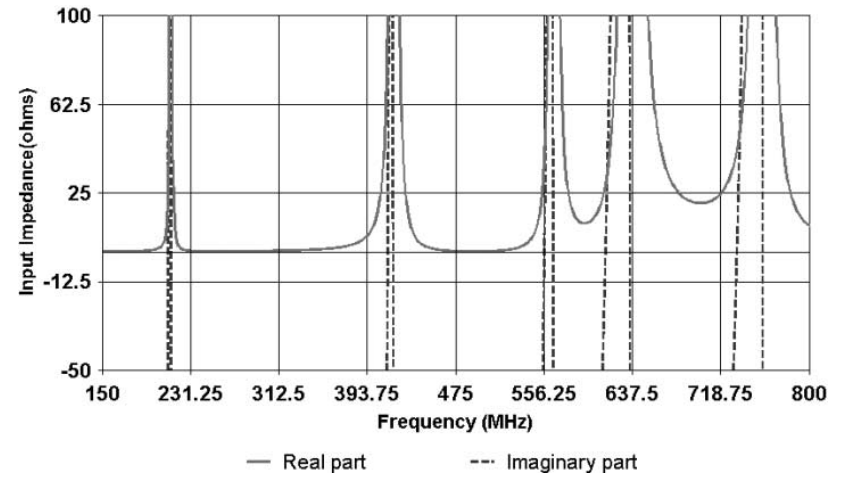

Fig. 5. Real and imaginary parts of the input impedance for the fifth iteration-order Hilbert antenna with feed point shown in Fig. 2. 
TABLE II

RESUlts of NEC SimUlation. HeRE $f_{0}$ IS THE FIRST RESONANT FREQUENCY AND BW IS THE VSWR BANDWIDTH (VSWR $<2$ WITH 50- $\Omega$ LINE)

\begin{tabular}{c|c|c|c|c|c}
\hline $\mathrm{n}$ & 1 & 2 & 3 & 4 & 5 \\
\hline $\mathrm{f}_{0}(\mathrm{MHz})$ & 717 & 496.6 & 351.75 & 261.3 & 211.16 \\
\hline $\mathrm{BW}(\%)$ & 4 & 0.94 & 0.31 & 0.11 & 0.05 \\
\hline
\end{tabular}

For the third iteration-order Hilbert antenna, we see some of the salient features in Fig. 3. We see that the current magnitude [Fig. 3(a)] is almost symmetric, and the phase [Fig. 3(b)] varies slowly along the wire length mainly due to the effect of mutual coupling among various line segments of this antenna. We note that the real part of input impedance is approximately $50 \Omega$ with a VSWR $=1.1$ at the fundamental resonant frequency. The bandwidth over which VSWR $<2$, however, is about $0.31 \%$. The radiation patterns of this antenna at this resonant frequency in the planes of $\phi=0^{\circ}$ and $\phi=90^{\circ}$ [Fig. 3(e)], almost resemble the patterns of a linear dipole, as was also pointed out by Vinoy et al. in [3].

Fig. 4 shows the properties of the fifth iteration-order matched Hilbert antenna. In this case, the VSWR bandwidth (with respect to $50-\Omega$ line) is about $0.05 \%$, which is even smaller than that of the third-order Hilbert antenna. Here, in addition to the current distribution, input impedance, and the VSWR, in Fig. 4(f) and (g) we show the color-coded contour plots of the magnitude and phase of current distribution along the wire length of this antenna. We again notice that the phase of the current varies slowly along the wire length. As expected, the multiresonant feature of this matched antenna with the feed point given in Fig. 2 can be seen from Fig. 5, where the real and imaginary parts of the input impedance for the fifth-order Hilbert antenna are plotted versus frequency. One can see that the input impedance is approximately $50 \Omega$ at the lowest and higher resonant frequencies.

It is clear that the plane-filling nature of Hilbert antennas makes them resonant at much lower frequencies compared with same-size patch antennas. In other words, for a Hilbert antenna occupying a fixed square surface area of $L \times L$, increasing $n$ from 1 to 5 significantly lowers the resonant frequency by a factor of three and higher. This reduction in the resonant frequency, however, is achieved at the expense of reduction of the bandwidth over which VSWR $<2$ (for a 50- $\Omega$ line). The first resonant frequency and such bandwidth for Hilbert antennas of iteration orders 1 through 5 with fixed side dimensions 70 $\mathrm{mm} \times 70 \mathrm{~mm}$ are listed in Table II. We notice that the fifth iteration-order Hilbert antenna can have $211.16 \mathrm{MHz}$ as the first resonant frequency. For the same size patch antenna, the resonant frequency would be $2.14 \mathrm{GHz}$. On the other hand, if one had wanted to have a patch antenna resonant at $211.16 \mathrm{MHz}$, the size of the patch would have been $710.35 \mathrm{~mm} \times 710.35 \mathrm{~mm}$.

Another important issue to consider in practical applications is the level of cross polarization in these antennas. For the matched Hilbert antennas with various iteration orders, this quantity is listed in Table III, in the planes $\phi=0^{\circ}$ and $\phi=90^{\circ}$. The electric fields, $E_{\theta}$ and $E_{\phi}$, in these two planes
TABLE III

LEVEl of CRosS POLARIZATION (dB)

\begin{tabular}{c|c|c|c|c|c|c}
\hline \multicolumn{2}{c|}{$n$} & 1 & 2 & 3 & 4 & 5 \\
\hline \multirow{2}{*}{$\begin{array}{c}\text { Level of } \\
\text { Cross - Pol } \\
\text { (dB) }\end{array}$} & $\begin{array}{c}\text { Phi= } \\
0\end{array}$ & -4.4 & -6.9 & -9.5 & -10.7 & -12.6 \\
\cline { 2 - 7 } & $\begin{array}{c}\text { Phi= } \\
90\end{array}$ & -58.9 & -56.3 & -59.3 & -58.5 & -58.5 \\
\hline
\end{tabular}

for a third-order Hilbert antenna are shown in Fig. 3(e). It is worth noting that if the magnitude and phase of the current distribution on this antenna had been exactly symmetric with respect to the plane of symmetry $\phi=90^{\circ}$, the level of cross polarization in this plane would have been $-\infty \mathrm{dB}$. However, due to the small variation in the phase of the current and the small deviation in the symmetry of the magnitude of the current, some level of cross polarization is present in the plane $\phi=90^{\circ}$. In the plane $\phi=0^{\circ}$, the cross-polarization level decreases as the iteration order is increased.

\section{CONCLUSION}

In this letter, the bandwidth, cross-polarization level, and feed-point location to achieve $50-\Omega$ input impedance at the fundamental frequency for the Hilbert antennas have been investigated using a moment-method based simulation code. The input impedance, VSWR bandwidth, magnitude and phase distributions of induced current along the wire, radiation patterns and cross-polarization levels of the matched Hilbert antennas have been presented. For fixed-area $50-\Omega$ matched Hilbert antennas, the first resonant frequency is lowered by increasing the iteration order $n$. However, the bandwidth is narrowed for the higher iteration orders, while the cross-polarization level is decreased in the nonsymmetrical plane of the antenna, i.e., the plane $\phi=0^{\circ}$.

\section{REFERENCES}

[1] H. Sagan, Space-Filling Curves. Berlin, Germany: Springer-Verlag, 1994.

[2] E. E. Altshuler, "Electrically small self-resonant wire antennas optimized using a genetic algorithm," IEEE Trans. Antennas Propagat., vol. 50, pp. 297-300, Mar. 2002.

[3] K. J. Vinoy, K. A. Jose, V. K. Varadan, and V. V. Varadan, "Hilbert curve fractal antenna: A small resonant antenna for VHF/UHF applications," Micrawave Opt. Technol. Lett., vol. 29, no. 4, pp. 215-219, May 2001.

[4] — , "Resonant frequency of Hilbert curve fractal antennas," in Proc. Dig. 2001 IEEE Antennas and Propagation Society Int. Symp., Boston, MA, July 2001, pp. 648-651.

[5] - "Hilbert curve fractal antennas with reconfigurable characteristics," in Proc. Dig. 2001 IEEE Microwave Theory and Techniques Society Int. Microwave Symp., vol. 3, Phoenix, AZ, May 2001, pp. 381-384.

[6] S. R. Best, "A comparison of the performance properties of the Hilbert curve fractal and meander line monopole antennas," Microwave Opt. Technol. Lett., vol. 35, no. 4, pp. 258-262, Nov. 2002.

[7] J. Romeu and S. Blanch, "A three-dimensional Hilbert antenna," in Proc. Dig. 2002 IEEE Antennas and Propagation Society Int. Symp., vol. 4, San Antonio, TX, June 2002, pp. 550-553.

[8] J. Anguera, C. Puente, and J. Soler, "Miniature monopole antenna based on fractal Hilbert curve," in Proc. Dig. 2002 IEEE Antennas and Propagation Society Int. Symp., vol. 4, San Antonio, TX, June 2002, pp. 546-549. 